\title{
Extragalactic Background Light, MACHOs, and the Cosmic Stellar Baryon Budget
}

\author{
Piero Madau \\ Department of Astronomy and Astrophysics, University of California, \\ Santa Cruz, CA 95064, USA \\ Francesco Haardt \\ Dipartimento di Scienze, Universitá dell'Insubria, via Lucini 3, Como, \\ Italy \\ Lucia Pozzetti \\ Osservatorio Astronomico di Bologna, Via Ranzani 1, 40127 Bologna, \\ Italy
}

\begin{abstract}
The optical/far-IR extragalactic background light (EBL) from both resolved and unresolved extragalactic sources is an indicator of the total luminosity of cosmic structures, as the cumulative emission from young and evolved galactic systems, as well as from active galactic nuclei (AGNs), is recorded in this radiation. This is a brief review of some of the implications of the observed brightness of the night sky for the stellar mass density and average metallicity of the universe today, and of the possible contribution of MACHO progenitors and QSOs to the EBL. Assuming a Salpeter initial mass function with a cutoff below $0.6 \mathrm{M}_{\odot}$, a lower limit of $\Omega_{g+s} h^{2}>0.0015 I_{60}$ can be derived to the visible (recycled gas + stars) mass density required to generate an EBL at a level of $I_{\mathrm{EBL}}=60 I_{60} \mathrm{nW} \mathrm{m}^{-2} \mathrm{sr}^{-1}$. Our latest, 'best-guess' estimate is $\Omega_{g+s} h^{2} \approx 0.0023 I_{60}$, which implies a mean metallicity at the present-epoch of $y_{Z} \Omega_{g+s} / \Omega_{b} \approx 0.2 Z_{\odot}$. If massive dark halos around spiral galaxies are partially composed of faint, old white dwarfs, i.e., if a non-negligible fraction ( a few percent) of the nucleosynthetic baryons is locked in the remnants (MACHOs) of intermediate-mass stars forming at very high redshifts, then the bright early phases of such halos should contribute significantly to the observed EBL. Assuming a standard black hole accretion model for quasar activity and using recent observations of the quasar population and new synthesis models for the cosmic $\mathrm{X}$ ray background, we estimate a present mass density of QSO remnants of $\rho_{\mathrm{BH}} \approx 3 \times 10^{5} \mathrm{M}_{\odot} \mathrm{Mpc}^{-3}$ for a $10 \%$ efficiency of accreted mass-toradiation conversion. The quasar contribution to the brightness of the night sky is $I_{\mathrm{QSO}} \approx 2 \mathrm{nW} \mathrm{m}^{-2} \mathrm{sr}^{-1}$.
\end{abstract}




\section{Introduction}

Observational studies of the distant universe are undergoing a revolution brought about by breakthroughs achieved with the HST, Keck, and JCMT telescopes, and the ISO satellite. The remarkable progress in our understanding of faint galaxy data made possible by the combination of HST deep imaging and groundbased spectroscopy has permitted us in the last few years to shed new light on the evolution of the stellar birthrate in the universe, to identify the epoch where most of the optical extragalactic background light was produced, and to set important constraints on galaxy evolution scenarios. The use of novel instruments and observational techniques has led to the measurement of the abundance and clustering of actively star-forming objects at redshift $3-4$ and to the discovery of galaxies and QSOs at redshift in excess of 5 , when the universe was less than $6 \%$ of its current age.

The explosion in the quantity of information available on the high-redshift universe at optical wavelengths has been complemented by the detection by DIRBE and FIRAS onboard the COBE satellite of the far-IR/sub-mm background - which has revealed that a significant fraction of the energy released by stellar nucleosynthesis is re-emitted as thermal radiation by dust - and by theoretical progress made in understanding how baryons follow the dynamics dictated by dark matter halos until radiative, hydrodynamic, and star formation processes take over. While ongoing studies with Chandra and XMM-Newton may be discovering a new population of highly absorbed, dusty AGNs (Type 2 QSOs), other recent findings of importance for galaxy formation models include a strong link between the masses of black holes in the nuclei of nearby galaxies and the properties of the host stellar bulges, and the suggestion from Magellanic Cloud microlensing experiments and systematic proper-motion surveys that a non-negligible fraction of the dark matter in the Galactic halo may be tied up in very old, cool white dwarfs. The underlying goal of all these efforts is to understand the growth of structures, the internal properties of galaxies and their evolution, and ultimately to map the star formation and supermassive black hole accretion histories of the universe from the end of the 'dark age' to the present epoch. The implications of the observed energy density of the EBL for the cosmic stellar and metal budget, and the quasar contribution to the brightness of the night sky, will be the subject of this talk. Unless otherwise stated, an Einstein-de Sitter (EdS) cosmology with $\Omega_{M}=1, \Omega_{\Lambda}=0$, and $H_{0}=100 \mathrm{~h} \mathrm{~km} \mathrm{~s}^{-1} \mathrm{Mpc}^{-1}$ will be adopted in the following.

\section{The Brightness of the Night Sky}

The logarithmic slope of the galaxy number-magnitude relation is a remarkably simple cosmological probe of the history of stellar birth in galaxies, as it must drop below 0.4 to yield a finite value for the EBL. This appears to be the case in all seven $U B V I J H K$ optical bandpasses, i.e., the light from resolved galaxies has converged from the UV to the near-IR (Madau \& Pozzetti 2000, hereafter MP). The flattening at faint apparent magnitudes cannot be due to the reddening of distant sources as their Lyman break gets redshifted into the blue passband, since the fraction of Lyman-break galaxies at (say) $B \approx 25$ 
is small. Moreover, an absorption-induced loss of sources cannot explain the similar change of slope of the galaxy counts observed in the $V, I, J, H$, and $K$ bands. While this suggests that the surface density of optically luminous galaxies is leveling off beyond $z \sim 1.5$, one should note that different algorithms used for 'growing' the photometry beyond the outer isophotes of galaxies may significantly change the magnitude of faint objects. According to Bernstein, Freedman, \& Madore (2000), roughly $50 \%$ of the flux from resolved galaxies with $V>23$ mag lies outside the standard-sized apertures used by photometric packages. An extragalactic sky pedestal created by the overlapping wings of resolved galaxies may contribute to the sky level, and would be undetectable except by absolute surface photometry. Also, at faint magnitude levels, distant objects which are brighter than the nominal depth of the catalog may be missed due to the $(1+z)^{4}$ dimming factor. All these systematic errors are inherent in faint-galaxy photometry; as a result, estimates of the integrated fluxes from resolved galaxies should be strictly considered as lower limits (see Pozzetti \& Madau, this volume).

The spectrum of the optical EBL is shown in Figure 1, together with the recent results from $C O B E$. The value derived by integrating the galaxy counts down to very faint magnitude levels (because of the flattening of the numbermagnitude relation most of the contribution to the optical EBL comes from relatively bright galaxies) implies a lower limit to the EBL intensity in the $0.2-$ $2.2 \mu \mathrm{m}$ interval of $I_{\mathrm{opt}} \approx 15 \mathrm{nW} \mathrm{m}^{-2} \mathrm{sr}^{-1}$. Including the tentative detections at 2.2 and $3.5 \mu \mathrm{m}$ by Gorjian, Wright, \& Chary (2000) (see also Dwek \& Arendt 1998) would boost $I_{\mathrm{opt}}$ to $\gtrsim 20 \mathrm{nW} \mathrm{m} \mathrm{sr}^{-2} \mathrm{sr}^{-1}$. Recent direct measurements of the EBL at 3000,5500 , and $8000 \AA$ from absolute surface photometry lie between a factor of 2.5 to 3 higher than the integrated light from galaxy counts, with an uncertainty that is largely due to systematic rather than statistical error (Bernstein et al. 2000). Applying this correction factor to the range 3000-8000 $\AA$ gives a total optical EBL intensity in the range $25-30 \mathrm{nW} \mathrm{m}^{-2} \mathrm{sr}^{-1}$. This could become $\sim 45 \mathrm{nW} \mathrm{m}^{-2} \mathrm{sr}^{-1}$ if the same correction holds also in the nearIR (Gorjian et al. 2000). The COBE/FIRAS (Fixsen et al. 1998) measurements yield $I_{\mathrm{FIR}} \approx 14 \mathrm{nW} \mathrm{m}^{-2} \mathrm{sr}^{-1}$ in the $125-2000 \mu \mathrm{m}$ range. When combined with the DIRBE (Hauser et al. 1998) points at 140 and $240 \mu \mathrm{m}$, one gets a far-IR background intensity of $I_{\text {FIR }}(140-2000 \mu \mathrm{m}) \approx 20 \mathrm{nW} \mathrm{m}^{-2} \mathrm{sr}^{-1}$. The detection with DIRBE of a FIR signal in excess of the expected zodiacal and Galactic emission by Finkbeiner et al. (2000), if confirmed, would imply an integrated EBL in the window $45-2000 \mu \mathrm{m}$ of $\gtrsim 40 \mathrm{nW} \mathrm{m}^{-2} \mathrm{sr}^{-1}$. The residual emission in the 3.5 to $140 \mu \mathrm{m}$ region is poorly known, but it is likely to exceed $10 \mathrm{nW} \mathrm{m}^{-2} \mathrm{sr}^{-1}$ (Dwek et al. 1998). to be

A 'best-guess' estimate of the total EBL intensity observed today appears

$$
I_{\mathrm{EBL}}=60 \pm 20 \mathrm{nW} \mathrm{m}^{-2} \mathrm{sr}^{-1}
$$

In the following, we will adopt a reference value for the background light associated with star formation activity over the entire history of the universe of $I_{\mathrm{EBL}}=60 I_{60} \mathrm{nW} \mathrm{m}^{-2} \mathrm{sr}^{-1}$. 


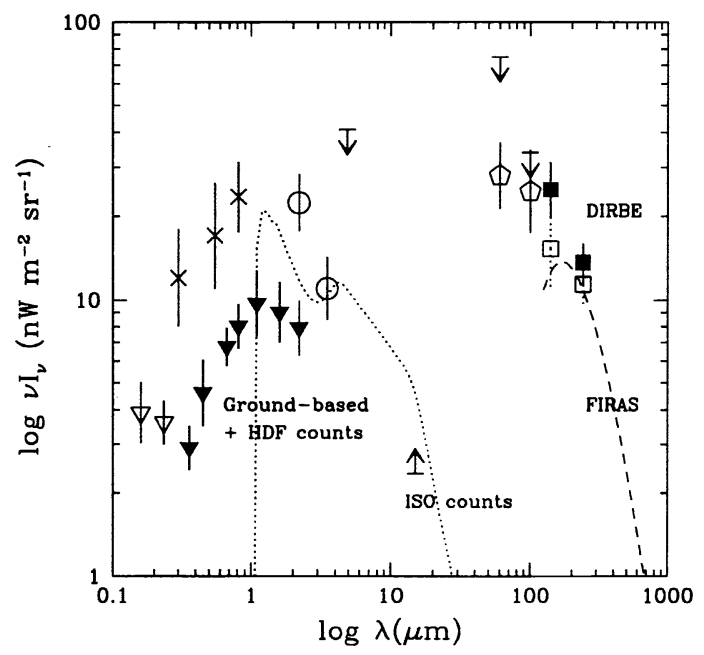

Figure 1. Spectrum of the optical extragalactic background light from resolved sources as derived from a compilation of ground-based and space-based galaxy counts in the UBVIJHK bands (filled triangles, MP), together with the FIRAS $125-5000 \mu \mathrm{m}$ (dashed line, Fixsen et al. 1998) and DIRBE 140 and $240 \mu \mathrm{m}$ (filled squares, Hauser et al. 1998) detections. The empty squares show the DIRBE points after correction for WIM dust emission (Lagache et al. 1999). Also plotted (empty triangles) are the STIS NUV (2360 $)$ and FUV (1600 $\AA$ ) points from Gardner et al. (2000), together with the tentative detections at 2.2 and $3.5 \mu \mathrm{m}$ (empty circles, Gorjian et al. (2000) and at 60 and $100 \mu \mathrm{m}$ (empty pentagons, Finkbeiner et al. 2000) from COBE/DIRBE observations. The crosses at 3000,5500 , and $8000 \AA$ are Bernstein et al. (2000) measurements of the EBL from resolved and unresolved galaxies fainter than $V=23 \mathrm{mag}$ (the error bars showing $2 \sigma$ statistical errors). Upper limits are from Hauser et al. (1998), the lower limit from Elbaz et al. (1999) ISO counts. The dotted curve shows the synthetic EBL produced by a WD-progenitor dominated IMF with $\bar{m}=4$ and $\left(z_{F}, X, X_{\mathrm{WD}}\right)=(11,0.2,0.04)$, in the case of zero dust reddening. 


\section{The Stellar Mass Density Today}

With the help of some simple stellar population synthesis tools we can now set a lower limit to the total stellar mass density that produced the observed EBL, and constrain the cosmic history of star birth in galaxies (MP; Harwit 1999; Madau, Pozzetti, \& Dickinson 1998): One of the most serious uncertainties in this calculation is the lower cutoff, usually treated as a free parameter, of the initial mass function (IMF). Observations of $\mathrm{M}$ subdwarfs stars with the HST have recently shed some light on this issue, showing that the IMF in the Galactic disk can be represented analytically over the mass range $0.1<m<1.6$ (here $m$ is in solar units) by $\log \phi(m)=$ const $-2.33 \log m-1.82(\log m)^{2}$ (Gould, Bahcall, \& Flynn 1996, hereafter GBF). For $m>1$ this mass distribution agrees well with a Salpeter function. A shallow mass function below $1 M_{\odot}$ has also been recently measured in the Galactic bulge (Zoccali et al. 2000) and in globular clusters (Paresce \& De Marchi 2000). Observations of normal Galactic starforming regions also show some convergence in the basic form of the IMF at intermediate and high masses, a power-law slope that is consistent with the Salpeter value (Elmegreen 1998; Massey 1998). In the following we will use a 'universal' IMF (shown in Figure 2) with the GBF form for $m<1$, matched to a Salpeter slope for $m \geq 1$; the mass integral of this function is 1.7 times smaller than that obtained by extrapolating a Salpeter function down to $0.1 M_{\odot}{ }^{1}$

As shown in Figure 3, the bolometric luminosity as a function of age $\tau$ of a simple stellar population (a single generation of coeval, chemically homogeneous stars having total mass $M$, solar metallicity, and the above IMF) can be well approximated by

$$
L(\tau)= \begin{cases}1200 L_{\odot} \frac{M}{M_{\odot}} & \tau \leq 2.6 \mathrm{Myr} \\ 0.7 L_{\odot} \frac{M}{M_{\odot}}\left(\frac{\tau}{1 \mathrm{Gyr}}\right)^{-1.25} & 2.6 \leq \tau \leq 100 \mathrm{Myr} \\ 2.0 L_{\odot} \frac{M}{M_{\odot}}\left(\frac{\tau}{1 \mathrm{Gyr}}\right)^{-0.8} & \tau>100 \mathrm{Myr}\end{cases}
$$

Over a timescale of $13 \mathrm{Gyr}$ - the age of the universe for an EdS cosmology with $h=0.5$ - about $1.3 \mathrm{MeV}$ per stellar baryon will be radiated away. This number depends only weakly on the assumed metallicity of stars. In a stellar system with arbitrary star formation rate per comoving cosmological volume, $\dot{\rho}_{s}$, the bolometric emissivity at time $t$ is given by the convolution integral

$$
\rho_{\text {bol }}(t)=\int_{0}^{t} L(\tau) \dot{\rho}_{s}(t-\tau) d \tau .
$$

The total background light observed at Earth $\left(t=t_{H}\right)$, generated by a stellar population with a formation epoch $t_{F}$, is

$$
I_{\mathrm{EBL}}=\frac{c}{4 \pi} \int_{t_{F}}^{t_{H}} \frac{\rho_{\mathrm{bol}}(t)}{1+z} d t
$$

\footnotetext{
${ }^{1}$ Since the bolometric light contributed by stars less massive than $1 M_{\odot}$ is very small for a 'typical' IMF, the use of a GBF mass function at low masses instead of Salpeter leaves the total radiated luminosity of a stellar population virtually unaffected.
} 


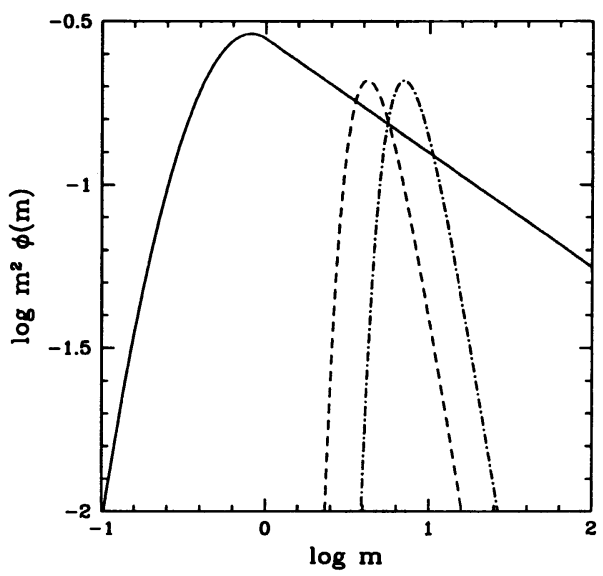

Figure 2. Stellar initial mass functions $\phi(m)$ (times $\left.m^{2}\right)$. Solid line: Salpeter IMF, $\phi(m) \propto m^{-2.35}$ at high masses, matched to a GBF function at $m \leq 1$ (see text for details). Dashed line: WD-progenitor dominated IMF in galaxy halos, $\phi(m) \propto e^{-(\bar{m} / m)^{3}} m^{-5}$, with $\bar{m}=2.4$ (see text for details). Dot-dashed line: Same for $\bar{m}=4$. All IMFs have been normalized to $\int m \phi(m) d m=1$.

where the factor $(1+z)$ in the denominator is lost to cosmic expansion when converting from observed to radiated (comoving) luminosity density.

To set a lower limit to the present-day mass density, $\Omega_{g+s}$, of recycled gas + stars (in units of the critical density $\rho_{\text {crit }}=2.8 \times 10^{11} h^{2} \mathrm{M}_{\odot} \mathrm{Mpc}^{-3}$ ), consider now a scenario where all stars are formed instantaneously at redshift $z_{F}$. The background light that would be observed at Earth from such an event is shown in Figure 3 as a function of $z_{F}$ for $\Omega_{g+s} h^{2}=0.0008,0.0013,0.0018$, corresponding respectively to 4,7 , and 9 percent of the nucleosynthetic baryon density, $\Omega_{b} h^{2}=0.0193 \pm 0.0014$ (Burles \& Tytler 1998). Two main results are worth stressing here: (1) the time evolution of the luminosity radiated by a simple stellar population (eq. 2) makes the dependence of the observed EBL from $z_{F}$ much shallower than the $\left(1+z_{F}\right)^{-1}$ lost to cosmic expansion (see eq. 4 ), as the energy output from stars is spread over their respective lifetimes; and (2) in order to generate an EBL at a level of $60 I_{60} \mathrm{nW} \mathrm{m}^{-2} \mathrm{sr}^{-1}$, one requires $\Omega_{g+s} h^{2}>0.0015 I_{60}$ for an EdS universe with $h=0.5$, hence a mean massto-blue light ratio today of $\left\langle M / L_{B}\right\rangle_{g+s}>4.1 I_{60}$ (the total blue luminosity density at the present-epoch is $\mathcal{L}_{B}=2 \times 10^{8} h \mathrm{~L}_{\odot} \mathrm{Mpc}^{-3}$, Ellis et al. 1996). The dependence of these estimates on the cosmological model (through eq. 4) is rather weak.

A visible mass density at the level of the above lower limit, while able to explain the measured sky brightness, requires all the stars that give origin to the observed EBL to have formed at very low redshifts $\left(z_{F} \lesssim 0.5\right)$, a scenario which 

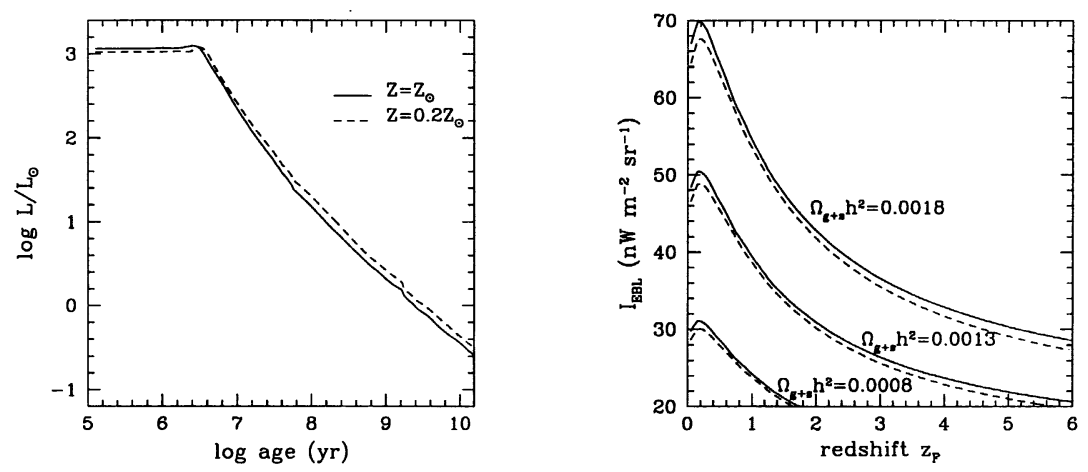

Figure 3. Left: Synthetic (based on an update of Bruzual \& Charlot's 1993 libraries) bolometric luminosity versus age of a simple stellar population having total mass $M=1 M_{\odot}$, metallicity $Z=Z_{\odot}$ (solid line) and $Z=0.2 Z_{\odot}$ (dashed line), and a GBF+Salpeter IMF (see text for details). Right: EBL observed at Earth from the instantaneous formation at redshift $z_{F}$ of the same stellar population $\left(Z=Z_{\odot}\right.$ case $)$ with total mass density $\Omega_{g+s} h^{2}=0.0018,0.0013$, and 0.0008. Solid curves: EdS universe with $h=0.5\left(t_{H}=13 \mathrm{Gyr}\right)$. Dashed curves: $\Lambda_{-}$ dominated universe with $\Omega_{M}=0.3, \Omega_{\Lambda}=0.7$, and $h=0.65\left(t_{H}=14.5\right.$ Gyr).

appears to be at variance with the observed evolution of the UV luminosity density (Lilly et al. 1996; Madau et al. 1998). For illustrative purposes, it is interesting to consider instead a model where the star formation rate per unit comoving volume stays constant with cosmic time. In an EdS cosmology with $h=0.5$, one derives from equations (2), (3), and (4)

$$
I_{\mathrm{EBL}}=1460 \mathrm{nW} \mathrm{m}^{-2} \mathrm{sr}^{-1}\left\langle\frac{\dot{\rho}_{s}}{\mathrm{M}_{\odot} \mathrm{yr}^{-1} \mathrm{Mpc}^{-3}}\right\rangle .
$$

The observed EBL therefore implies a 'fiducial' mean star formation rate (SFR) density of $\left\langle\dot{\rho}_{s}\right\rangle=0.04 I_{60} \quad \mathrm{M}_{\odot} \mathrm{yr}^{-1} \mathrm{Mpc}^{-3}$, a factor 3 higher than the value measured at $\langle z\rangle=0.15$ by Treyer et al. (1998). ${ }^{2}$ Any value much smaller than this over a sizeable fraction of the Hubble time will generate an EBL well short of $60 \mathrm{nW} \mathrm{m}{ }^{-2} \mathrm{sr}^{-1}$. Ignoring for the moment the recycling of returned gas into new stars, the visible mass density at the present epoch is simply $\rho_{g+s}=$ $\int_{0}^{t_{H}} \dot{\rho}_{s}(t) d t=5.2 \times 10^{8} I_{60} \mathrm{M}_{\odot} \mathrm{Mpc}^{-3}$, corresponding to $\Omega_{g+s} h^{2}=0.0019 I_{60}$ and $\left\langle M / L_{B}\right\rangle_{g+s}=5.2 I_{60}$ (both values would be a factor of 1.7 higher in the case of a Salpeter IMF down to $\left.0.1 M_{\odot}\right)$.

\footnotetext{
${ }^{2}$ For the Treyer et al. data we have used the conversion from UV luminosity density to SFR per unit volume, $\log \dot{\rho}_{s}=\log \mathcal{L}_{2000}-28.1$, appropriate for the assumed IMF, and corrected upwards the observed luminosity density $\mathcal{L}_{2000}$ by a factor of 1.8 for dust extinction.
} 
A more realistic scenario which fits the most recent measurements of the rest-frame UV-continuum and $\mathrm{H} \alpha$ luminosity densities from the present-epoch to $z=4$ (after a correction for dust extinction is applied to the data) and produces a total EBL of about the right magnitude $\left(I_{60}=1\right)$, is one where the SFR density evolves as (EdS, $h=0.5$ )

$$
\dot{\rho}_{s}(z)=\frac{0.1 e^{2.2 z}}{e^{2.2 z}+6} \mathrm{M}_{\odot} \mathrm{yr}^{-1} \mathrm{Mpc}^{-3} .
$$

This SFR density (an updated version of the one used in eq. 8 of MP) increases slowly - by about a factor of 4 - from the present-epoch to $z=1$ (cf. Cowie et al. 1999) and remains constant at $z>2$ (Steidel et al. 1999). Since about half of the present-day stars are formed at $z>1.5$ in this model and their contribution to the EBL is redshifted away, the resulting visible mass density is $\Omega_{g+s} h^{2}=0.0023 I_{60}$ and $\left\langle M / L_{B}\right\rangle_{g+s}=6.3 I_{60}$, slightly larger than in the $\dot{\rho}_{s}=$ const approximation.

We conclude that, depending on the star formation history and for the assumed IMF, the observed EBL requires between $8 \%$ and $12 \%$ of the nucleosynthetic baryon density to be today in the forms of stars, recycled gas, and their remnants. According to the most recent census of cosmic baryons, the mass density in stars and their remnants observed today is $\Omega_{s} h=0.00245_{-0.00088}^{+0.00125}$ (Fukugita, Hogan, \& Peebles 1998), corresponding to a mean stellar mass-toblue light ratio of $\left\langle M / L_{B}\right\rangle_{s}=3.4_{-1.3}^{+1.7}$ (roughly $70 \%$ of this mass is found in old spheroidal populations). While this is lower than the $\left\langle M / L_{B}\right\rangle_{g+s}$ ratio predicted by equation (6), one should note that efficient recycling of ejected gas into new star formation would tend to reduce the apparent difference in the budgets. With the adopted IMF, about $30 \%$ of this mass will be returned to the interstellar medium in $10^{8} \mathrm{yr}$, after intermediate-mass stars eject their envelopes and massive stars explode as supernovae. This return fraction, $R$, becomes $50 \%$ after about $10 \mathrm{Gyr}^{3}$ Alternatively, the gas returned by stars may be ejected into the intergalactic medium. With an IMF-averaged yield of returned metals of $y_{Z} \approx 1.5 Z_{\odot},{ }^{4}$ the predicted mean metallicity at the present epoch is $y_{Z} \Omega_{g+s} / \Omega_{b} \approx 0.2 Z_{\odot}$, similar to the values inferred from cluster abundances (Renzini 1997).

The cosmic stellar baryon and metal budget is summarized in Table 1 for the three different modes (instantaneous, constant, and evolving) of SF considered. Note that a steeper IMF - e.g., a Scalo function which is significantly less rich in massive stars than Salpeter - or an IMF which does not flatten below $0.6 \mathrm{M}_{\odot}$ would generate mass-to-light ratios that are too high compared to the observed values.

${ }^{3} \mathrm{An}$ asymptotic mass fraction of stars returned as gas, $R=\int\left(m-m_{f}\right) \phi(m) d m \times$ $\left[\int m \phi(m) d m\right]^{-1} \approx 0.5$, can be obtained by using the semiempirical initial $(m)$-final $\left(m_{f}\right)$ mass relation of Weidemann (1987) for stars with $1<m<10$, and by assuming that stars with $m>10$ return all but a $1.4 \mathrm{M}_{\odot}$ remnant.

${ }^{4}$ Here we have taken $y_{z} \equiv \int m p_{\mathrm{zm}} \phi(m) d m \times\left[\int m \phi(m) d m\right]^{-1}$, the stellar yields $p_{\mathrm{zm}}$ of Tsujimoto et al. (1995), and a GBF+Salpeter IMF. In the case of a Salpeter IMF down to $0.1 \mathrm{M}_{\odot}, y_{Z}$ should be multiplied by 0.6 . 


\begin{tabular}{cccc}
\hline \hline SF mode & $\Omega_{g+s} h^{2}$ & $\left\langle M / L_{B}\right\rangle_{g+s}$ & $y_{Z} \Omega_{g+s} / \Omega_{b}$ \\
\hline instantaneous & 0.0015 & 4.1 & 0.12 \\
constant & 0.0019 & 5.2 & 0.15 \\
evolving & 0.0023 & 6.3 & 0.18 \\
\hline
\end{tabular}

Table 1. The Cosmic Stellar Baryon and Metal Budget. These values assume an EdS cosmology with $h=0.5$, a GBF+Salpeter IMF, and an EBL intensity of $60 \mathrm{nW} \mathrm{m}^{-2} \mathrm{sr}^{-1}$.

\section{EBL from MACHOs}

The nature of the dark matter in the halo of galaxies remains one of the outstanding problems in astrophysics. One of the most interesting constraints posed by the observed brightness of the night sky concerns the possibility that a significant fraction of the dark mass in present-day galaxy halos may be associated with faint white-dwarf (WD) remnants of a population of intermediate-mass stars that formed at high redshifts. The latest results of the microlensing MA$\mathrm{CHO}$ experiment towards the LMC, interpreted in the context of a Galactic dark matter halo consisting partially of compact objects, ${ }^{5}$ give a MACHO halo fraction of $20 \%$, with a $95 \%$ confidence interval of 8-50\% (Alcock et al. 2000). Similar results have been reached by the EROS collaboration (Lasserre et al. 2000). The most likely MACHO mass is between 0.15 and $0.9 \mathrm{M}_{\odot}$ : this mass scale is a natural one for WDs, a scenario also supported by the lack of a numerous spheroidal population of low-mass main sequence stars in the HDF (Gould et al. 1998), and by the recent discovery of two ancient halo WDs in a systematic proper motion survey (Ibata et al. 2000). The total mass of MACHOs inferred within $50 \mathrm{kpc}$ is $9_{-3}^{+4} \times 10^{10} M_{\odot}$ (Alcock et al. 2000), implying a 'MACHO-toblue light' ratio for the Milky Way in the range 4 to 10 solar (cf Fields, Freese, $\&$ Graff 1998). If these values were typical of the luminous universe as a whole, i.e., if MACHOs could be viewed as a new stellar population having similar properties in all disk galaxies, then the cosmological mass density of MACHOs today would be $\Omega_{\mathrm{MACHO}}=(4-10) f_{B} \mathcal{L}_{B} / \rho_{\text {crit }}=(0.003-0.007) f_{B} h^{-1}$, a significant entry in the cosmic baryon budget, $\Omega_{\mathrm{MACHO}} / \Omega_{b}=(0.15-0.4) f_{B} h$. Here $f_{B} \approx 0.5$ is the fraction of the blue luminosity density radiated by stellar disks (Fukugita et al. 1998). Note that if MACHOs are halo WDs, the contribution of their progenitors to the mass density parameter is several times higher.

Halo IMFs which are very different from that of the solar neighborhood, i.e., which are heavily-biased towards WD progenitors and have very few stars forming with masses below $2 M_{\odot}$ (as these would produce bright WDs in the halo today that are not seen) and above $8 M_{\odot}$ (to avoid the overproduction of heavy elements), have been suggested as a suitable mechanism for explaining the microlensing data (Chabrier et al. 1996). While the halo WD scenario may

\footnotetext{
${ }^{5}$ Another widely discussed possibility is that microlensing is actually dominated by self-lensing in a LMC halo.
} 
be tightly constrained by the observed rate of Type Ia SNe in galaxies (Smecker \& Wyse 1991), by the expected C and N overenrichment of halo stars (Gibson \& Mould 1997), and by the number counts of faint galaxies in deep optical surveys (Charlot \& Silk 1995), here we explore a potentially more direct method (as it does not depend on, e.g., extrapolating stellar yields to primordial metallicities, on galactic winds removing the excess heavy elements into the intergalactic medium, or on the reddening of distant halos by dust), namely we will compute the contribution of WD progenitors in dark galaxy halos to the extragalactic background light.

Following Chabrier (1999) (see also MP), we adopt a truncated power-law IMF,

$$
\phi(m)=\text { const } \times e^{-(\bar{m} / m)^{3}} m^{-5} .
$$

This form mimics a mass function strongly peaked at $0.84 \bar{m}$. To examine the dependence of the IMF on the results we consider two functions (shown in Figure 2), $\bar{m}=2.4$ and $\bar{m}=4$ : both yield a present-day Galactic halo massto-light ratio $>100$ after a Hubble time, as required in the absence of a large non-baryonic component. We further assume that a population of halo WD progenitors having mass density $X \Omega_{b} h^{2}=0.0193 X$ formed instantaneously at redshift $z_{F}$ with this IMF and nearly primordial $\left(Z=0.02 Z_{\odot}\right)$ metallicity. The resulting EBL from such an event is shown in Figure 4 for $X=0.1,0.3$, and 0.6 and a $\Lambda$-dominated universe with $\Omega_{M}=0.3, \Omega_{\Lambda}=0.7$, and $h=0.65\left(t_{H}=14.5\right.$ Gyr).

Consider the $\bar{m}=2.4$ case first. With $z_{F}=3$ and $X=0.6$, this scenario would generate an EBL at a level of $300 \mathrm{nW} \mathrm{m}^{-2} \mathrm{sr}^{-1}$. Even if only $30 \%$ of the nucleosynthetic baryons formed at $z_{F}=5$ with a WD-progenitor dominated IMF, the resulting background light at Earth would exceed the value of $100 \mathrm{nW} \mathrm{m}^{-2} \mathrm{sr}^{-1}$, the 'best-guess' upper limit to the observed EBL from the data plotted in Figure 1 . Since the return fraction of this IMF is $R \approx 0.8$, only $20 \%$ of this stellar mass would be left over as WDs, the rest being returned to the ISM. Therefore, if galaxy halos comprise $100 \%$ of the nucleosynthetic baryons, only a small fraction of their mass, $X_{\mathrm{WD}} \approx 0.2 \times 0.3=0.06$, could be in the form of WDs. ${ }^{6}$

In qualitative agreement with the most recent microlensing results (Alcock et al. 2000; Lasserre et al. 2000), these limits likely imply a non-baryonic dark halo. On the other hand, we draw attention to the fact that, even if only $20 \%$ of the baryons in the universe turned into halo WD progenitors at $z_{F} \gtrsim 10$, their background light should be detectable as a peak in the EBL around $1 \mu \mathrm{m}$. In Figure 1 we show the EBL produced by such a WD-progenitor dominated IMF with $\bar{m}=4$ and $\left(z_{F}, X, X_{\mathrm{WD}}\right)=(11,0.2,0.04)$, assuming negligible dust reddening. Intriguingly, this model appears to be consistent with measurements of the optical/near-IR EBL by Bernstein et al. (2000) and Gorjian et al. (2000).

\footnotetext{
${ }^{6}$ Pushing the peak of the IMF to more massive stars, $\bar{m}=4$, helps only marginally. With $\bar{m}=2.4$, the energy radiated per stellar baryon over a timescale of $13 \mathrm{Gyr}$ is equal to $2 \mathrm{MeV}$, corresponding to $10 \mathrm{MeV}$ per baryon in WD remnants. A similar value is obtained in the $\bar{m}=4$ case: because of the shorter lifetimes of more massive stars the expected EBL is reduced, but only by $20 \%$ or so (see Figure 4 ). Moreover, the decreasing fraction of leftover WDs would raise more severe problems of metal galactic enrichment.
} 

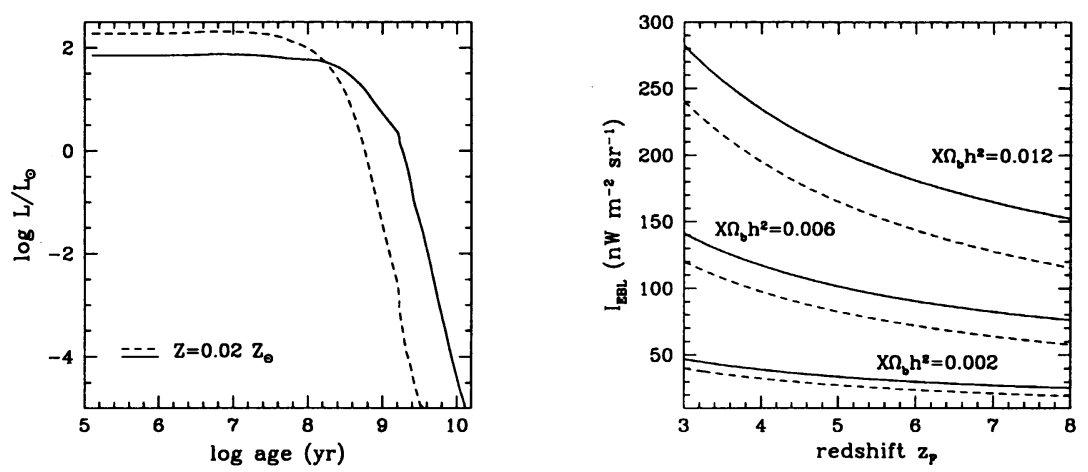

Figure 4. Left: Synthetic bolometric luminosity versus age of a simple stellar population having total mass $M=1 M_{\odot}$, metallicity $Z=0.02 Z_{\odot}$, and a WD-progenitor dominated IMF (see text for details) with $\bar{m}=2.4$ (solid line) and $\bar{m}=4$ (dashed line). Right: $\mathrm{EBL}$ observed at Earth from the instantaneous formation at redshift $z_{F}$ of a stellar population having the same IMF and metallicity, and mass density $X \Omega_{b} h^{2}=0.012,0.006$ and 0.002 (corresponding to 60,30 , and 10 per cent of the nucleosynthetic value of Burles \& Tytler 1998), as a function of $z_{F}$. A $\Lambda$-dominated universe with $\Omega_{M}=0.3, \Omega_{\Lambda}=0.7$, and $h=0.65$ has been assumed. Solid line: $\bar{m}=2.4$. Dashed line: $\bar{m}=4$.

\section{EBL from Quasar Activity}

An accurate determination of the contribution of QSOs to the EBL, $I_{\mathrm{QSO}}$, obtainable in principle by integrating the number-magnitude relation down to the detection threshold, is in practice made uncertain by our poor knowledge of the mean bolometric correction and of the sky brightness due to undetected faint objects. Such a direct method also explicitly neglects the possible existence of a population of dusty AGNs with strong intrinsic absorption as invoked, e.g., in many models for the X-ray background (e.g., Madau, Ghisellini, \& Fabian 1994; Comastri et al. 1995): these Type II QSOs, while undetected at optical wavelengths, could contribute significantly to the far-IR background and to the total EBL. Moreover, the conversion of $I_{\mathrm{QSO}}$ to the expected mass density of quasar remnants at the present-epoch, $\rho_{\mathrm{BH}}$, (assuming a standard black hole accretion model for QSOs) requires a correction for the radiation energy lost to the cosmic expansion which depends on the quasar redshift distribution.

Some (but hardly all) of these difficulties can perhaps be bypassed by computing instead, in analogy with the stellar case,

$$
I_{\mathrm{QSO}}=\frac{c}{4 \pi} \int_{0}^{t_{H}} \frac{d t}{1+z} \int_{0}^{\infty} L \phi(L, t) d L
$$



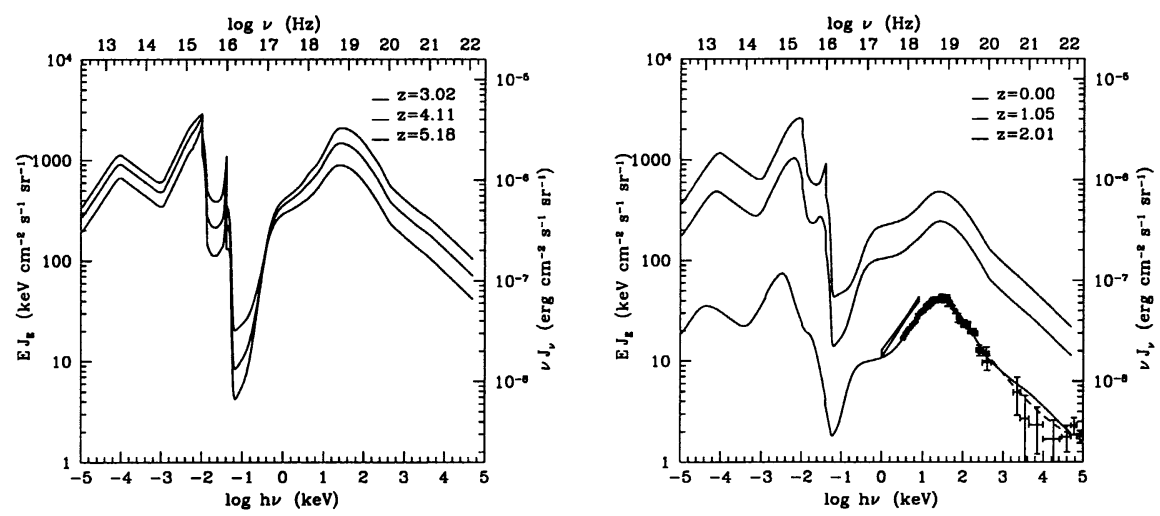

Figure 5. Synthetic radiation background from quasars including the reprocessing of UV radiation from intervening absorption systems. Left: metagalactic flux at $z=5.18,4.11$ and 3.02 (from top to bottom above $10^{17} \mathrm{~Hz}$ ). Right: same at $z=2.01,1.05$ and 0 (from top to bottom). The data points at $z=0$ show the spectrum of the observed $\mathrm{X}$ and $\gamma$-ray background. (From Haardt \& Madau 2000.)

where $\phi(L, t)$ is the comoving bolometric luminosity function of QSOs at cosmic time $t$. We have modeled $\phi(L, t)$ adopting up-to-date determinations of the quasar number density and spectral energy distribution from the far-IR to gamma-rays, and using new synthesis models for the cosmic X-ray background (XRB) based on a mixture of unabsorbed Type I and heavily absorbed Type II AGNs (Haardt \& Madau 2000). The spectrum of the radiation background from QSOs is shown in Figure 5 (we indicate it with $J$ instead of $I$ as it has been filtered through the absorption of the intergalactic medium at UV frequencies, cf. Haardt \& Madau 1996) as a function of redshift. Our model can simultaneously reproduce the observed spectrum of the XRB and the most recent source counts in both the soft and hard X-ray bands, and yields at the present-epoch a value of $I_{\mathrm{QSO}} \approx 2 \mathrm{nW} \mathrm{m} \mathrm{sr}^{-1}$ (previous estimates range from 0.7 to $3 \mathrm{nW} \mathrm{m}^{-2} \mathrm{sr}^{-1}$, Soltan 1982; Chokshi \& Turner 1992; Small \& Blandford 1992). In determining the mass density of quasar remnants at the present-epoch we take into account the large contribution due to the absorbed radiation of Type II AGNs (Fabian \& Iwasawa 1999). Such radiation (which is not shown in the spectrum of Figure 5) is most probably re-emitted in the far IR band. We derive

$$
\rho_{\mathrm{BH}}=\frac{1}{c^{2} \eta} \int_{0}^{t_{H}} d t \int_{0}^{\infty} L \phi(L, t) d L \approx 3 \times 10^{4} \eta^{-1} \mathrm{M}_{\odot} \mathrm{Mpc}^{-3}
$$

where $\eta$ is the efficiency of accreted mass-to-radiation conversion, equal to $5.7 \%$ for standard disk accretion onto a Schwarzschild black hole.

It is in principle possible to gauge our model for the evolution and emission properties of the quasar population by weighing the local mass density of black holes remnants. Recent dynamical evidence indicates that supermassive black holes reside at the center of most nearby galaxies (Richstone et al. 1998). The 
available data (more than 30 objects) show an empirical correlation between bulge luminosity and black hole mass (Magorrian et al. 1998), which becomes even tighter when bulge velocity dispersion is plotted instead of luminosity (Ferrarese \& Merritt 2000; Gebhardt et al. 2000). The implied mean ratio of black hole to bulge mass is $M_{\mathrm{BH}} \approx 0.0013 M_{\mathrm{sph}}$ as a best-fit (Merritt \& Ferrarese 2000), a factor of 5 smaller than the mean ratio computed by Magorrian et al. (1998). The mass density in old spheroidal populations today is estimated to be $\Omega_{\mathrm{sph}} h=0.0018_{-0.00085}^{+0.0012}$ (Fukugita et al. 1998), implying a mean mass density of quasar remnants today of

$$
\rho_{\mathrm{BH}} \approx 6 \times 10^{5} h \mathrm{M}_{\odot} \mathrm{Mpc}^{-3} .
$$

This determination agrees with the result of equation (9) for $\eta \sim 0.05 h^{-1}$.

Acknowledgments. We thank the organizers of the IAU Symposium 204, M. Harwit and M. Hauser, for their patience in waiting for this manuscript, and T. Matsumoto for discussions on MACHO progenitors and the EBL. Support for this work was provided by NASA through ATP grant NAG5-4236 (P. M.), and by C.N.A.A. and ASI through contract ASI-ARS-98-119 (L. P.).

\section{References}

Alcock, C., et al. 2000, ApJ, 542, 281

Bernstein, R. A., Freedman, W. L., \& Madore, B. F. 2000, submitted

Bruzual, A. C., \& Charlot, S. 1993, ApJ, 405, 538

Burles, S., \& Tytler, D. 1998, ApJ, 499, 699

Chabrier, G. 1999, ApJ, 513, L103

Chabrier, G., Segretain, L., \& Mera, D. 1996, ApJ, 468, L21

Charlot, S., \& Silk, J. 1995, ApJ, 445, 124

Chokshi, A., \& Turner, E. L. 1992, MNRAS, 259, 421

Comastri, A., et al. 1995, A\&A, 296, 1

Cowie, L. L., Songaila, A., \& Barger, A. J. 1999, AJ, 118, 603

Dwek, E., \& Arendt, R. G. 1998, ApJ, 508, L9

Dwek, E., et al. 1998, ApJ, 508, 106

Elbaz, D., et al. 1999, A\&A, 351, L37

Ellis, R. S., Colless, M., Broadhurst, T., Heyl, J., \& Glazebrook, K. 1996, MNRAS, 280, 235

Elmegreen, B. G. 1998, in Unsolved Problems in Stellar Evolution, ed. M. Livio (Cambridge: Cambridge University Press), in press (astro-ph/9811289)

Fabian, A. C., \& Iwasawa, K. 1999, MNRAS, 303, L34

Ferrarese, L., \& Merritt, D. 2000, ApJ, 539, L9

Fields, B. D., Freese, K., \& Graff, D. S. 1998, NewA, 3, 347

Finkbeiner, D. P., Davis, M., \& Schlegel, D. J. 2000, ApJ, 544, 81

Fixsen, D. J., et al. 1998, ApJ, 508, 123 
Fukugita, M., Hogan, C. J., \& Peebles, P. J. E. 1998, ApJ, 503, 518

Gardner, J. P., Brown, T. M., \& Ferguson, H. C. 2000, ApJ, 542, L79

Gebhardt, K., et al. 2000, ApJ, 539, L13

Gibson, B., \& Mould, J. 1997, ApJ, 482, 98

Gorjian, V., Wright, E. L., \& Chary, R. R. 2000, ApJ, 536, 500

Gould, A., Bahcall, J. N., \& Flynn, C. 1996, ApJ, 465, 759 (GBF)

Gould, A., Flynn, C., \& Bahcall, J. N. 1998, ApJ, 503, 798

Haardt, F., \& Madau, P. 1996, ApJ, 461, 20

Haardt, F., \& Madau, P. 2000, in preparation

Harwit, M. 1999, ApJ, 510, L83

Hauser, M. G., et al. 1998, ApJ, 508, 25

Ibata, R., Irwin, M., Bienaymé, O., Scholz, R., \& Guibert, J. 2000, ApJ, 532, L41

Lagache, G., Abergel, A., Boulanger, F., Desert, F. X., \& Puget, J.-L. 1999, $\mathrm{A} \& \mathrm{~A}, 344,322$

Lasserre, T., et al. 2000, A\&A, 355, L39

Lilly, S. J., Le Févre, O., Hammer, F., \& Crampton, D., 1996, ApJ, 460, L1

Madau, P., Ghisellini, G., \& Fabian, A. C. 1994, MNRAS, 270, L17

Madau, P., \& Pozzetti, L. 2000, MNRAS, 312, L9 (MP)

Madau, P., Pozzetti, L., \& Dickinson, M. 1998, ApJ, 498, 106

Magorrian, G., et al. 1998, AJ, 115, 2285

Massey, P. 1998, in The Stellar Initial Mass Function, ed. G. Gilmore \& D. Howell (San Francisco: ASP), 17

Merritt, D., \& Ferrarese, L. 2000, preprint (astro-ph/0009076)

Paresce, F., \& De Marchi, G. 2000, ApJ, 534, 870

Renzini, A. 1997, ApJ, 488, 35

Richstone, D., et al. 1998, Nature, 395, 14

Small, T. A., \& Blandford, R. D. 1992, MNRAS, 259, 725

Smecker, T. A., \& Wyse, R. 1991, ApJ, 372, 448

Soltan, A. 1982, MNRAS, 200, 115

Steidel, C. C., Adelberger, K. L., Giavalisco, M., Dickinson, M., \& Pettini, M. 1999, ApJ, 519, 1

Treyer, M. A., Ellis, R. S., Millard, B., Donas, J., \& Bridges, T. J. 1998, MNRAS, 300,303

Tsujimoto, T., Nomoto, K., Yoshii, Y., Hashimoto, M., Yanagida, S., \& Thielemann, F.-K. 1995, MNRAS, 277, 945

Weidemann, V. 1987, A\&A, 188, 74

Zoccali, M., et al. 2000, ApJ, 530, 418 